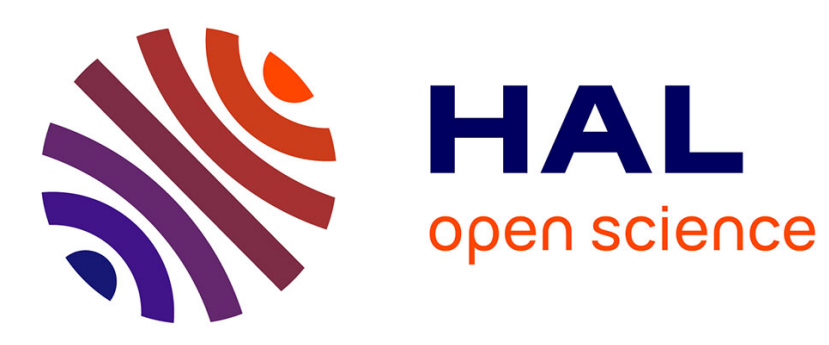

\title{
Involvement of the human midbrain and thalamus in auditory deviance detection
}

\author{
Raffaele Cacciaglia, Carles Escera, Lavinia Slabu, Sabine Grimm, Ana \\ Sanjuán, Noelia Ventura-Campos, César Ávila
}

\section{To cite this version:}

Raffaele Cacciaglia, Carles Escera, Lavinia Slabu, Sabine Grimm, Ana Sanjuán, et al.. Involvement of the human midbrain and thalamus in auditory deviance detection. Neuropsychologia, 2015, 68, pp.51 - 58. 10.1016/j.neuropsychologia.2015.01.001 . hal-01753380

\section{HAL Id: hal-01753380 \\ https://hal.science/hal-01753380}

Submitted on 6 May 2018

HAL is a multi-disciplinary open access archive for the deposit and dissemination of scientific research documents, whether they are published or not. The documents may come from teaching and research institutions in France or abroad, or from public or private research centers.
L'archive ouverte pluridisciplinaire HAL, est destinée au dépôt et à la diffusion de documents scientifiques de niveau recherche, publiés ou non, émanant des établissements d'enseignement et de recherche français ou étrangers, des laboratoires publics ou privés. 


\title{
Involvement of the human midbrain and thalamus in auditory deviance detection
}

\author{
Raffaele Cacciaglia ${ }^{\mathrm{a}, \mathrm{b}}$, Carles Escera ${ }^{\mathrm{a}, \mathrm{b}, \mathrm{n}}$, Lavinia Slabu ${ }^{\mathrm{a}, \mathrm{b}}$, Sabine Grimm ${ }^{\mathrm{a}, \mathrm{b}}$, Ana Sanjuán ${ }^{\mathrm{c}, \mathrm{d}}$, Noelia \\ Ventura-Campos ${ }^{c}$, César Ávila ${ }^{\mathrm{c}}$ \\ ${ }^{\text {a }}$ Institute for Brain, Cognition and Behavior (IR3C), University of Barcelona, Passeig de la vall d'Hebron 171, 08035 Barcelona, Catalonia, Spain \\ ${ }^{\mathrm{b}}$ Cognitive Neuroscience Research Group, Department of Psychiatry and Clinical Psychobiology, University of Barcelona, Passeig de la vall d'Hebron 171, \\ 08035 Barcelona, Catalonia, Spain \\ ${ }^{\mathrm{c}}$ Department of Psychology, University Jaume I, Avenguda de Vicent Sos Baynat, 12071 Castellón de la Plana, Spain \\ ${ }^{\mathrm{d}}$ Language Group Wellcome Trust Centre for Neuroimaging University College of London, 12 Queen Square, WC1N 3BG London, United Kingdom
}

Corresponding author at: Cognitive Neuroscience Research Group, Department of Psychiatry and Clinical Psychobiology, University of Barcelona. Passeig de la Vall d'Hebron 171, 08035 Barcelona, Catalonia, Spain. Fax: p34 934021 584. E-mail address: cescera@ub.edu (C. Escera)

\begin{abstract}
Prompt detection of unexpected changes in the sensory environment is critical for survival. In the auditory domain, the occurrence of a rare stimulus triggers a cascade of neurophysiological events spanning over multiple time-scales. Besides the role of the mismatch negativity (MMN), whose cortical generators are located in supratemporal areas, cumulative evidence suggests that violations of auditory regularities can be detected earlier and lower in the auditory hierarchy. Recent human scalp recordings have shown signatures of auditory mismatch responses at shorter latencies than those of the MMN. Moreover, animal single-unit recordings have demonstrated that rare stimulus changes cause a release from stimulusspecific adaptation in neurons of the primary auditory cortex, the medial geniculate body (MGB), and the inferior colliculus (IC). Although these data suggest that change detection is a pervasive property of the auditory system which may reside upstream cortical sites, direct evidence for the involvement of subcortical stages in the human auditory novelty system is lacking. Using eventrelated functional magnetic resonance imaging during a frequency oddball paradigm, we here report that auditory deviance detection occurs in the MGB and the IC of healthy human participants. By implementing a random condition controlling for neural refractoriness effects, we show that auditory change detection in these subcortical stations involves the encoding of statistical regularities from the acoustic input. These results provide the first direct evidence of the existence of multiple mismatch detectors nested at different levels along the human ascending auditory pathway.
\end{abstract}

Keywords: Auditory deviance detection, Stimulus-specific adaptation fMRI, Inferior colliculus, Medial geniculate body

\section{Introduction}

In order to detect relevant information, our sensory systems continuously monitor the external environment. This is particularly true for the auditory system, which receives time-varying signals that are transient in nature and thus require to be tracked over time. To achieve this, the auditory system automatically extracts statistical regularities from the ongoing acoustic input and generates predictions enabling to detect unexpected deviant sounds (Bendixen et al., 2012; Winkler et al., 2009). Such automatic change detection promotes the formation of meaningful auditory objects (Bizley and Cohen, 2013; Griffiths and Warren, 2004) and drives a rapid attention shift towards potentially relevant stimuli (Escera et al., 1998; Escera and Corral, 2007). Auditory deviance detection has traditionally been associated to a particular brain event occurring at 150-250 ms from change onset, the Mismatch Negativity (MMN) of the event-related potentials (ERPs) (Näätänen et al., 1978, 2007), generated in supratemporal (Recasens et al., 2014; Maess et al., 2007) and prefrontal areas (Deouell, 2007; Rinne et al., 2000).

Yet, a convergent body of evidence suggests that regularity violations can be detected earlier and in lower stations of the auditory hierarchy (Escera and Malmierca, 2014). Indeed, mismatch responses for simple deviant features have been observed at latencies much shorter than those of MMN, in different components of the middle latency response (MLR) of the human ERPs, peaking at 18-50 ms from stimulus onset (Escera et al., 2014; Grimm et al., 2011, 2012; Slabu et al., 2010a; Sonnadara et al., 2006), and in the complex auditory brainstem response (Slabu et al., 2012), suggesting the involvement of the human inferior colliculus (IC) in deviance detection. Animal single- and multiunit recordings have shown that neurons in the primary auditory cortex of the cat (Ulanovsky et al., 2003), and in the medial geniculate body (MGB) and IC of the rat (Antunes and Malmierca, 2011; Malmierca et al., 2009; PérezGonzález et al., 2005), display stimulus-specific adaptation (SSA), a reduction in neuronal firing rates for repetitive sounds, that is restored for rare stimuli. Moreover, a recent study employing functional magnetic resonance imaging
(fMRI) showed that the IC significantly responded to rarely occurring auditory stimuli in rats (Gao et al., 2014). These animal and human data favor the idea of deviance detection being a ubiquitous and hierarchically organized property of the auditory pathway (Grimm and Escera, 2012). Yet the involvement of the human subcortical auditory system in deviance detection remains to be proven. Using event-related fMRI during a frequency oddball paradigm, we here report that auditory deviance detection occurs in the human auditory cortex, the MGB and the IC.

The underlying neural mechanisms of the MMN and more generally of auditory change detection are not yet fully understood. Two major theoretical accounts have been proposed within the past years: a former model that puts forward the formation of an auditory sensory memory based on the encoding of statistical regularities from the acoustic input (Näätänen et al., 2005), and a complementary view that ascribes deviance detection to a mechanism of release from neural adaptation (May and Tiitinen, 2010). Here, by implementing a random condition controlling for stimulus probability and the related neural refractoriness effects (cfr., Schröger and Wolff, 1996), we show that change detection in the MGB and IC is likely based on a mechanism of regularity encoding and therefore cannot be explained by mere adaptation phenomena.

\section{Materials and methods}

\subsection{Subjects}

Twelve volunteer participants $(7$ female, mean age $=27.5$ years, standard deviation $[\mathrm{SD}]=3.4,4$ left-handed) took part in our experiment. None of them was under current or chronic medication. All participants had normal hearing, with a mean hearing threshold below $25 \mathrm{~dB}$ sound pressure level (dB-SPL), as assessed with binaural audiometric test using pure tones at five frequencies $(250,500,1000,3000$, and $8000 \mathrm{~Hz})$. The experimental protocol was approved by 
Ethical Committee of the University of Barcelona and was in accordance with the Code of Ethics of the World Medical Association (Declaration of Helsinki). Written informed consent was obtained before the experiment.

\subsection{Stimuli}

Stimuli consisted of $70 \mathrm{~ms}$ bursts of broadband noises with no rise and fall was the same of the SOA $(150 \mathrm{~ms})$. One-hundred-twenty-five trains each containing 4 deviant and 16 standard stimuli $\left(40 \mathrm{DEV}_{1}, 40 \mathrm{DEV}_{2}\right.$ and 45 $\mathrm{DEV}_{3}$ ) were randomly interleaved with 125 trains containing 20 standards stimuli only (STD condition). In this way, a single functional volume could be acquired for each train (see Image Acquisition section). Thereafter, a control condition $(\mathrm{CON})$, consisting of 125 consecutively presented trains of 20 stimuli was administered. In each train, five different stimuli (S1, S2, S3,

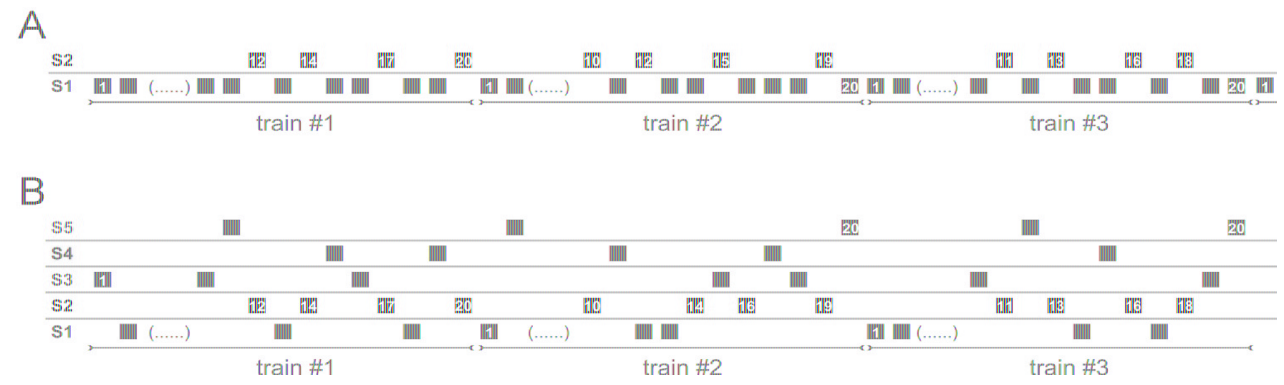

Fig. 2. Experimental paradigm. (A) The oddball paradigm consisted of 125 trains containing 20 identical stimuli acting as standard (i.e. train \#4), randomly interleaved with 125 train containing the standard plus the deviant stimulus (i.e. trains \#1, \#2, \#3) (B) The control condition consisted of 125 trains each containing five randomly distributed and equiprobable stimuli presented with the same timing parameters as in the oddball paradigm, with the only constrain that S2 occurred in the same physical position as it was in the oddball paradigm, where it acted as deviant.

time, which were generated with the STIM software (Neurosoft, El Paso, TX, USA). They were band-pass filtered in steps of $500 \mathrm{~Hz}$, from 500 to $1000 \mathrm{~Hz}$ (referred as S1), from 1000 to $1500 \mathrm{~Hz}$ (referred as S2), from 1500 to $2000 \mathrm{~Hz}$ (referred as S3), from 2000 to $2500 \mathrm{~Hz}$ (referred as S4) and from 2500 to 3000 $\mathrm{Hz}$ (referred as S5) (Fig. 1). These stimuli were chosen on the base of a previous study in which they elicited reliable auditory brainstem responses (Slabu et al., 2010a). All stimuli were binaurally delivered at a constant stimulus onset asynchrony (SOA) of $150 \mathrm{~ms}$ through a MR-compatible headphone set which attenuates scanner noise by $15 \mathrm{~dB}$ (VisuaStim digital, Resonance Technology Inc., Northridge, CA, USA). Intensity was individually calibrated as being $20 \%$ above the discrimination level with respect to the scanner noise.

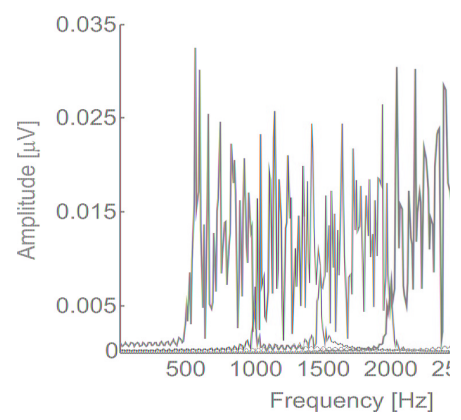

Fig. 1. Experimental stimuli. Amplitude spectrum of the five stimuli used in the experiment Frequency bands are color-coded as follows: S1 (black), S2 (red), S3 (cyan), S4 (green), S5 (orange). (For interpretation of the references to color in this figure legend, the reader is referred to the web version of this article.)

\subsection{Experimental design}

The experiment consisted of a passive frequency oddball paradigm, which was followed by a random condition controlling for neural refractoriness effects. In the oddball paradigm, two stimuli were used, one of which (S1) acted as standard, while the other (S2) as deviant. Deviant sounds had a probability of $20 \%$ and were embedded in trains of 20 stimuli, occurring at pseudorandom positions in the second half of the train, that is, between the tenth and the twentieth sound. Specifically, deviant sounds were placed at positions $12,14,17,20$, or $10,12,15,19$, or $11,13,16,18$, in separate trains (Fig. 2a). These grouping arrangements produced three different deviant conditions which will be referred as $\mathrm{DEV}_{1}, \mathrm{DEV}_{2}$, and $\mathrm{DEV}_{3}$, according to the positions occupied by $\mathrm{S} 2$. The inter-train interval
S4 and S5) were randomly arranged equiprobably, with the only constrain that the stimulus (S2) acting as deviant during the oddball paradigm, preserved the same physical position also in the control condition, following the same grouping arrangements as in the DEV condition (see above) (Fig. $2 \mathrm{~b}$ ). Thus, also the $\mathrm{CON}$ condition could be further subdivided in $\mathrm{CON}_{1}$, $\mathrm{CON}_{2}$, and $\mathrm{CON}_{3}$, each of these matching the respective $\mathrm{DEV}_{1}, \mathrm{DEV}_{2}$ and $\mathrm{DEV}_{3}$ for the physical position of S2. Such a random condition was firstly introduced by Schröger and Wolff (1996) in order to control for refractoriness effects, as neural populations responsive to the CON stimuli presumably reveal the same degree of refractoriness as neurons responsive to the DEV stimuli. A similar control condition was already successfully implemented in event-related fMRI studies (e.g., Opitz et al., 2005; Szycik et al., 2013), in human ERPs (e.g., Jacobsen and Schröger, 2003) and animal multi-unit recordings (e.g., Taaseh et al., 2011).

Participants lay inside the scanner throughout the experiment. They were instructed to ignore the sounds and to watch a silent subtitled movie, which was projected using VisuaStim XGA goggles (VisuaStim digital, Resonance Technology Inc., Northridge, CA, USA).

\subsection{Image acquisition}

The fMRI session was performed with a $1.5 \mathrm{~T}$ full body scanner (Magnetom Siemens AVANTO, Siemens Medical Solutions, Erlangen, Germany), equipped with an eight channel phased-array transmit/receive head coil. Blood oxygenation level-dependent (BOLD) contrast functional images were acquired using a $\mathrm{T}_{2}{ }^{*}$-weighted gradient-echo Echo Planar Imaging (EPI) sequence (echo time, $\mathrm{TE}=39 \mathrm{~ms}$, repetition time $\mathrm{TR}=3000 \mathrm{~ms}$, flip angle $=90^{\circ}$, field of view, FOV $=192 \times 256 \mathrm{~mm}^{3}$, matrix size $=96 \times 128$, voxel size $=2 \times 2 \times 2 \mathrm{~mm}^{2}$ ). Each image volume consisted of 32 contiguous, 2 mm-thick slices (no interslice gap) recorded in ascending order and positioned by forming an angle of $45^{\circ}$ with respect to the longitudinal axis of the brainstem. This slice orientation minimizes the heartbeat-related motion along the dorso-ventral and rostro-caudal axes of the brainstem (Slabu, 2010b). The recorded functional volume covered the brainstem, thalamic structures, basal ganglia, the temporal lobes, plus motor and prefrontal areas. In total, 375 scans were acquired: 125 for the trains containing standard stimuli only (STD condition), 125 for the trains containing standard plus deviant stimuli (DEV condition), and 125 for the respective trains containing five randomly arranged stimuli (CON condition). Prior to scanning, 3 dummy functional volumes were acquired 
and discarded in order to allow for $\mathrm{T}_{1}$ saturation effects. For anatomical reference, structural images were acquired using a $\mathrm{T}_{1}$-weighted highresolution 3D gradient echo pulse sequence $(\mathrm{TE}=4.94 \mathrm{~ms}$, $\mathrm{TR}=11 \mathrm{~ms}$, flip angle $=15^{\circ}$, matrix size $=256 \times 224 \times 176$, voxel size $=1 \times 1 \times 1 \mathrm{~mm}^{3}$ ).

\section{5. fMRI data analysis}

Pre-processing, single-subject and group analyzes were performed with Statistical Parametrical Mapping (SPM8, http://www. fil.ion.ucl.ac.uk/spm/), implemented in Matlab R2012a (The MathWorks Inc., Natick, MA, USA). Time-series were slice-time corrected to reference slice 16 for difference in acquisition timing and realigned with a two-pass procedure in which functional volumes were registered to the first volume in the series, and to the mean image of all the realigned volumes. None of the study participants exceeded motion estimates of $2 \mathrm{~mm}$ and 2 degrees. After realignment, images were co-registered with the individual structural volumes and normalized to the Montreal Neurological Institute International (MNI) Consortium for Brain Mapping space using DARTEL registration, a procedure which increases spatial accuracy when mapping activation into small brain regions (Ashburner, 2007). A DARTEL template was created by reiteratively registering all the individuals' structural $\mathrm{T}_{1}$-weighted images, which were previously segmented into grey and white matter using the new segmentation routine provided by SPM8. The generated flow fields were subsequently used to warp the coregistered time-series into an MNI standardized space. Finally, images were spatially smoothed with a $4 \mathrm{~mm}^{3}$ full-width at half-maximum (FWHM) Gaussian kernel. To remove low-frequency noise, a high-pass filter (cutoff $1 / 128 \mathrm{~Hz}$ ) was included and the time-series were corrected for serial autocorrelations using first-order autoregressive functions AR(1). At singlesubject level, a fixed effects analysis was conducted by setting up a general linear model (GLM) including the following experimental conditions: standard (STD), deviant (DEV), and control (CON), plus the six motion parameters generated during realignment. To control for physiological noise, a regressor containing the extracted mean time-series of cerebrospinal fluid (CSF) was included and modeled as variance of no interest. These inputs were convolved with a canonical hemodynamic response function (first order expansion) to form the design matrix. At group level, a whole brain random effects analysis was performed using a voxel-wise one-sample t-tests with the contrasts of interest (DEV $>\mathrm{STD}$; DEV $>\mathrm{CON})$. Results were considered significant at $\mathrm{P}<0.05$, cluster corrected for multiple testing using a family-wise error (FWE) rate approach, applying a primary threshold of $\mathrm{P}<0.001$ and a cluster extent threshold of 15 voxels, following recent recommendations (Woo et al., 2014). Further, data were analyzed with a region of interest (ROI) approach, using a small volume correction (SVC) as implemented in SPM8. For cortical areas, masks for the ROI analysis were defined based on previously reported brain regions involved in auditory deviance detection, and included the superior temporal gyrus (STG), the Heschl's gyrus (HG) and the inferior frontal gyrus (IFG) (Doeller et al., 2003; Opitz et al., 2002, 2005; Schall et al., 2003). These ROIs were generated using the MNI template Automated Anatomical Labeling (Tzourio-Mazoyer et al., 2002), implemented in the Wake Forest University Pick Atlas toolbox for SPM8 (Maldjian et al., 2003). Given its large cluster extent, the STG mask was further subdivided in an anterior ( $y>-15)$, middle ($35<y<15)$ and posterior $(y<-35)$ portion (cfr. Ischebecket al., 2008). Masks for the IC and MGB were defined by drawing a $5-\mathrm{mm}$ radius sphere centered around previously published standardized MNI coordinates [IC: $\pm 6,-33,-11$; MGB: $\pm 14,-24,-8$ ] (Mühlau et al., 2006; von Kriegstein et al., 2008). To provide a functional spatial layout in the entire brainstem and to control for other potential activated regions in this brain area, we additionally performed a ROI analysis using the midbrain mask provided by the AAL atlas. Results were considered significant at $\mathrm{P}<0.05$ corrected for multiple testing using a FWE correction. Percent signal change was computed with the MARSeille Boîte À Région d'Intérêt (Marsbar) toolbox for SPM8 (http://marsbar.sourceforge.net/).

\subsection{DEV versus STD}

Whole-brain analysis conducted for the DEV >STD contrast revealed significant activations in the right $\mathrm{STG}\left(\mathrm{t}_{11}=6.59, \mathrm{P}_{\mathrm{FWE}}=0.003, \mathrm{k}=31,[\mathrm{x}=62, \mathrm{y}=12, \mathrm{z}=6]\right)$, and in the left STG $\left(\mathrm{t}_{11}=6.89\right.$, P Fwe $\left.=0.008, \mathrm{k}=53,[\mathrm{x}=-54, \mathrm{y}=-28, \mathrm{z}=12]\right)$. ROI analysis yielded significant activations in the anterior $\left(t_{11}=6.59\right.$, PFwe $<0.011$, $k=35[x=62, y=-12, z=6])$, middle $\left(t_{11}=5.68, P_{F W E}<0.001, k=49,[x=60, y=-20\right.$, $\mathrm{z}=0]$ ), and posterior $\left(\mathrm{t}_{11}=4.09, \operatorname{PFWE}_{\mathrm{F}}<0.014, \mathrm{k}=22,[\mathrm{x}=54, \mathrm{y}=-38, \mathrm{z}=8]\right)$ portions of the right STG, plus the anterior $\left(\mathrm{t}_{11}=6.47, \mathrm{PFWE}_{\mathrm{F}}<0.015, \mathrm{k}=23,[\mathrm{x}=-60, \mathrm{y}=-14\right.$, $\mathrm{z}=6])$ and middle $\left(\mathrm{t}_{11}=6.89, \mathrm{P}_{\mathrm{FWE}}<0.012, \mathrm{k}=55,[\mathrm{x}=-54, \mathrm{y}=-28, \mathrm{z}=12]\right)$ portions of the left STG. These results held when using the entire STG mask (right STG: $\mathrm{t}_{11}=6.59, \quad P_{\mathrm{FWE}}<0.042, \mathrm{k}=55, \quad[\mathrm{x}=62, \mathrm{y}=-12, \mathrm{z}=6]$; left STG: $\mathrm{t}_{11}=6.82$, PFwe $=0.029, \mathrm{k}=61,[\mathrm{x}=-58, \mathrm{y}=-20, \mathrm{z}=8])$. Additionally, there was a significant activation in the left HG $\left(\mathrm{t}_{11}=5.81, \mathrm{PFWE}_{0}<0.026, \mathrm{k}=19,[\mathrm{x}=-40, \mathrm{y}=-24\right.$, $\mathrm{z}=10]$ )(Fig. 3a, b). No significant activation could be detected in the IFG. Analysis performed with subcortical ROIs yielded significant activations in the left IC ( $\left.\mathrm{t}_{11}=5.24, \mathrm{P}_{\mathrm{FWE}}=0.023, \mathrm{k}=33,[\mathrm{x}=6, \mathrm{y}=32, \mathrm{z}=14]\right)$ (Fig. 4a, e), as well as in the right MGB $\left(\mathrm{t}_{11}=5.41, \mathrm{P}_{\mathrm{FWE}}=0.018, \mathrm{k}=34,[\mathrm{x}=14, \mathrm{y}=26, \mathrm{z}=10]\right)$, and in the left MGB ( $t_{11}=7.22, P_{F W E}=0.003, k=48,[x=12, y=24, z=-12]$ ) (Fig. 4b, e). An additional ROI analysis performed with the entire midbrain mask did not reveal further significant activated regions located in this area.

\subsection{DEV versus CON}

For this contrast, a whole brain analysis did not reveal any brain regions surviving FWE correction. However, a ROI analysis yielded a significant activation in the anterior portion of the left $\mathrm{STG}\left(\mathrm{t}_{11}=5.41\right.$, PFwE $=0.048, \mathrm{k}=24$, $[x=48, y=4, z=14])$. Critically, we observed significant activations in the left IC t11. =4.73, $P_{\mathrm{FWE}}=0.037, \mathrm{k}=24,[\mathrm{x}=6, \mathrm{y}=30, \mathrm{z}=12]$ ) (Fig. 4c, e), as well as in the right MGB $\left(\mathrm{t}_{11}=4.24, \mathrm{P}_{\mathrm{FWE}}=0.041, \mathrm{k}=19,[\mathrm{x}=12, \mathrm{y}=26, \mathrm{z}=12]\right)$ and left MGB $\left(\mathrm{t}_{11}=4.21, \mathrm{P}_{\mathrm{FWE}}=0.042, \mathrm{k}=27,[\mathrm{x}=14, \mathrm{y}=-24, \mathrm{z}=-14)\right]$ (Fig. $\left.4 \mathrm{~d}, \mathrm{e}\right)$. No additional regions were significantly activated, as revealed by a ROI analysis performed using the midbrain mask. These control analyzes highlight the specificity of our findings and support the role of the human IC and MGB in genuine deviance detection, i.e., based on regularity encoding. Fig. 4e suggests that the hemodynamic response to the STD trains of stimuli was larger than that elicited by the CON trains, however a follow-up analysis involving the STD $>$ CON contrast did not reveal any significant nor trends to significant results (for details refer to the Supplementary Material).

To further substantiate our data, we performed a more stringent analysis, where both the DEV and the CON conditions were divided into three independent conditions, according to the position of S2 within each train (see Experimental Design). We then compared the BOLD response elicited by each of the three DEV conditions $\left(\mathrm{DEV}_{1}, \mathrm{DEV}_{2}, \mathrm{DEV}_{3}\right)$ to that triggered by the corresponding $\mathrm{CON}$ condition $\left(\mathrm{CON}_{1}, \mathrm{CON}_{2}, \mathrm{CON}_{3}\right)$. This analysis yielded significant activation in the IC and MGB in most of the contrasts of interest. More specifically, in $\mathrm{DEV}_{1}>\mathrm{CON}_{1}$ we found a significant response in the left IC $\left(\mathrm{t}_{11}=5.81, \mathrm{P}_{\mathrm{FWE}}=0.013, \mathrm{k}=44,[\mathrm{x}=-4, \mathrm{y}=-28, \mathrm{z}=-12]\right)$ and in the right MGB $\left(\mathrm{t}_{11}=5.29, \mathrm{P}_{\mathrm{FWE}}=0.017, \mathrm{k}=37,([\mathrm{x}=10, \mathrm{y}=-26, \mathrm{z}=-10])\right.$ significant activations the left MGB $\left(\mathrm{t}_{11}=7.10, \mathrm{P}_{\mathrm{FWE}}=0.004, \mathrm{k}=22,[\mathrm{x}=-12, \mathrm{y}=-24, \mathrm{z}=-\right.$ 12]). Finally, in $\mathrm{DEV}_{3}>\mathrm{CON}_{3}$, we found, significant activations in the left IC $\left(t_{11}=4.44, P_{F W E}=0.035, k=16,[x=-6, y=-32, z=-10]\right)$, the right $M G B\left(t_{11}=4.20\right.$, $\left.P_{\mathrm{FWE}}=0.047, \mathrm{k}=37, \quad[\mathrm{x}=14, \mathrm{y}=-24, \mathrm{z}=-10]\right)$ and left MGB $\left(\mathrm{t}_{11}=5.62\right.$, $\left.P_{F W E}=0.016, k=30,[x=-12, y=-24, z=-12]\right)$. These analyses, that implemented a more stringent control by taking into consideration the exact position of deviants, confirm the role of the IC and MGB in deviance detection based on regularity encoding.

\section{Discussion}


The results of this study represent the first direct evidence that auditory deviance detection based on regularity encoding in humans occurs in subcortical stations of the auditory pathway. Using event-related fMRI during a controlled oddball paradigm, we reported that auditory change is detected in the midbrain and auditory thalamus of healthy human participants. We investigated responses in a priori defined regions of interest, as suggested by previous functional imaging studies and animal electrophysiological recordings (cfr. Escera and Malmierca, 2014; Escera et al., 2014). Specifically, we found significant activations in the left IC and the bilateral MGB in response to deviant-containing trains of stimuli versus trains containing standard stimuli only. In addition, we found significant activations in the bilateral STG and left HG, matching previous imaging studies implementing frequency oddball tasks, where activation of these cortical regions has been consistently reported (Doeller et al., 2003; Opitz et al., 2002, 2005; Sabri et al., 2004; Schall et al., 2003).

To better investigate the process underlying deviance detection and to rule out a possible effect of solely adaptation, we included a condition controlling for stimulus probability and the related neural refractoriness effects, as already implemented in previous human ERP (Jacobsen and Schröger, 2003) and animal multi-unit recordings (Taaseh et al., 2011), as well as in fMRI studies (Opitz et al., 2005; Szycik et al., 2013). Remarkably, when comparing

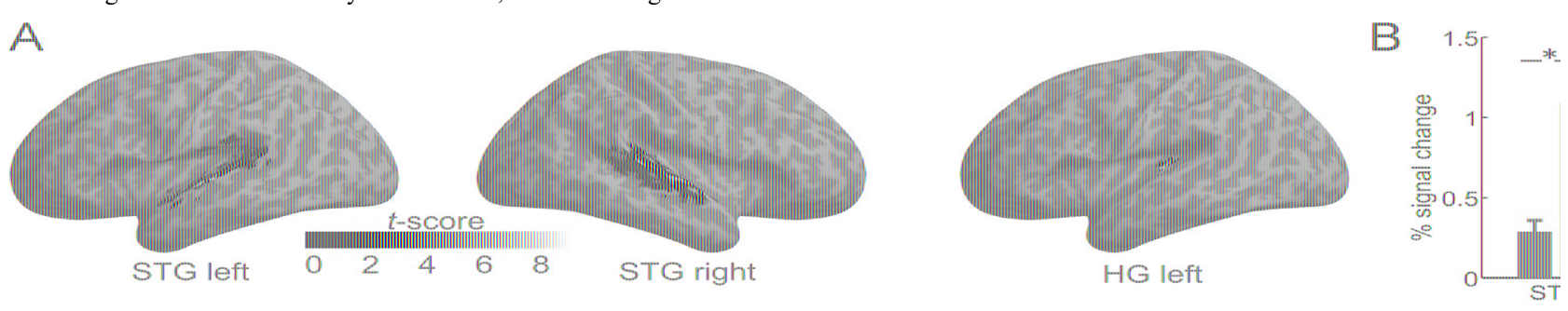

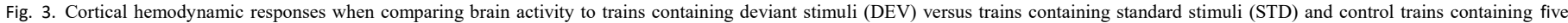

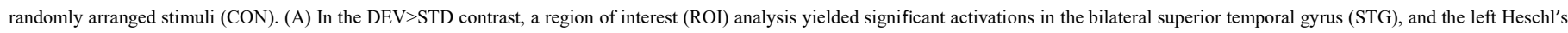

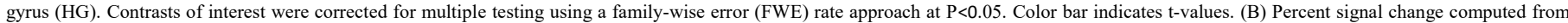

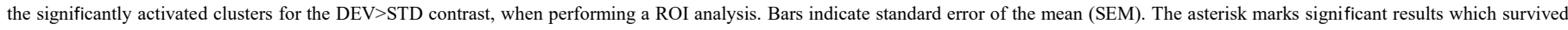
correction for multiple testing. (For interpretation of the references to color in this figure legend, the reader is referred to the web version of this article.)
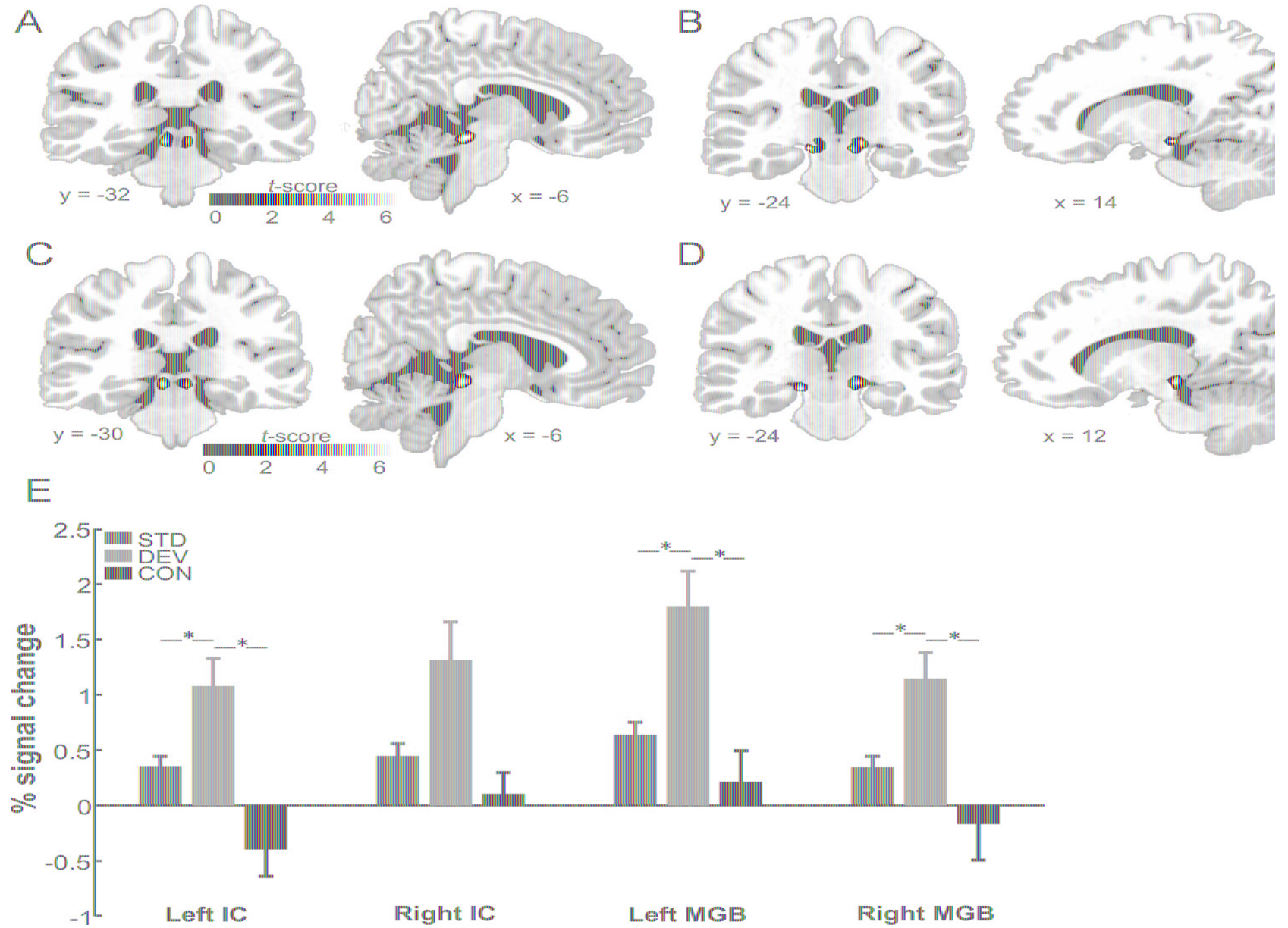

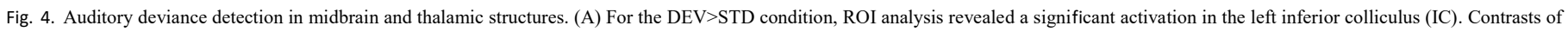

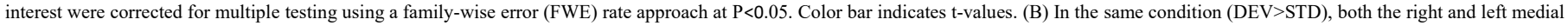

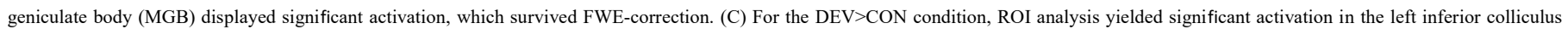

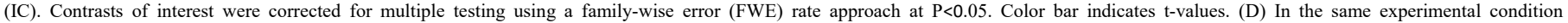

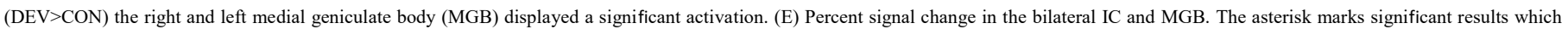

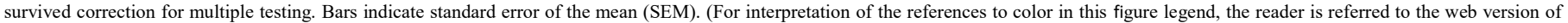
this article.) 
activity triggered by deviant-containing trains of stimuli to that elicited by stimuli in the control condition, we found significant brain responses in the same subcortical regions already observed for the DEV>STD contrast, that is the left IC and the bilateral MGB. Such a result is remarkable, because it indicates that auditory change detection in both the IC and MGB relied on the encoding of statistical regularities in addition to a possibly co-existing mechanism of release from adaptation, suggesting that a neural representation of the invariant aspect of the input is held early in the auditory hierarchy.

The IC is the major auditory nucleus of the midbrain and represents an obligatory relay station for all the ascending inputs. It receives two parallel systems of afferent projections: one directly coming from the controlateral cochlear nucleus, which supplies a rapid encoding of spectral features, and the other by both the ipsiand contro-lateral superior olivary complex, carrying information about localization, intensity and fine temporal structure of sounds (Winer, 2005). The IC is the first auditory structure where the organization in lemniscal (or primary) and non-lemniscal (or nonprimary) systems begins to appear, a morpho-functional segregation that holds for all the subsequent auditory structures (Lee and Sherman, 2011). Because of its strategic position, the IC serves as a neural hub that integrates spectral, temporal and other physical features of the sounds (i.e., intensity and location). On the other hand, the MGB is critical in relaying subcortical processed information to cortical areas, to which it directly projects with information coming from the IC.

So far, evidence accounting for a subcortical detection of acoustic change derived from electrophysiological recordings in animals. In their influential work, Kraus et al. (1994a) used intrathalamic recordings in the guinea pig during a frequency oddball paradigm, and found a significant evoked mismatch response in the non-primary subdivision of the auditory thalamus between 3080 and $130-175 \mathrm{~ms}$ from stimulus onset. The authors also implemented a "deviant alone" condition to control for refractoriness effects and could confirm an increased negativity appearing between $40-125 \mathrm{~ms}$ post stimulus in the nonprimary MGB, when comparing evoked responses to stimuli presented as rare in the oddball sequence versus physically identical stimuli presented alone. Comparable results have been reported for the processing of rarely occurring speech-like stimuli (Kraus et al., 1994b) and for sounds differing in their binaural phase (King et al., 1995).

Subsequent single-unit recording studies showed SSA in the medial subdivision of the MGB in mice (Anderson et al., 2009) as well as in the medial and dorsal MGB of anesthetized (Antunes et al., 2010; Antunes and Malmierca, 2011) and awake (Richardson et al., 2013) rats. Likewise, SSA has been reported in the dorsal aspect of the IC using local field potentials (LFPs) (Patel et al., 2012), and in single neurons throughout the subdivisions of the IC (Pérez-González et al., 2005; Malmierca et al., 2009). Recently, the involvement of the IC in deviance detection has been confirmed in rodents using BOLD fMRI (Gao et al., 2014). Thus, our results are in line with previously reported data in animals and extend those findings to the human subcortical auditory system.

It is worth noting that while we observed a strong signal in the IC in our contrasts of interest, no or little collicular SSA was found by others when the stimuli were delivered at a similar presentation rate used here (150-ms) (e.g., Malmierca et al., 2009). This might be due, in addition to species-specific differences, to the fact that the BOLD response captures synaptic activity over a large neuronal assembly, and therefore individual differences among neurons' responses are averaged out. This interpretation fits with the findings reported by Patel et al. (2012), who found the highest degree of collicular SSA when stimuli were presented at $8 \mathrm{~Hz}$ SOA using LFPs, an index of neuronal activity that the BOLD response is more closely linked to (Logothetis et al., 2001; Viswanathan and Freeman, 2007).

Besides the data obtained with animal intracranial recordings, evidence accounting for early auditory mismatch response have come from several human EEG studies conducted in our laboratory, where specific physical features of deviant stimuli were manipulated (cfr. Escera et al., 2014). For example, increased amplitude of the $\mathrm{Nb}$ evoked component was reported for a frequency change (Alho et al., 2012; Grimm et al., 2011; Leung et al., 2012), while enhanced $\mathrm{Na}$ amplitude was found for intensity (Althen et al., 2011) and location deviants (Grimm et al., 2012). Importantly, the majority of these studies implemented a random condition controlling for refractoriness confounds, thus indicating the presence of a "true" deviance detection at the level of the MLR. In line with earlier human EEG data, the results from the present study indicate that auditory deviance detection represents a basic principle of the functional organization of the ascending auditory pathway. The lack of significant activation in the right IC for both contrasts of interests is unlikely due to hearing difficulties in our participants, given that all of them reported normal audiometry data, and sound intensity was individually calibrated. It is possible that, the implementation in our paradigm of an auditory attentional task would yield stronger collicular activation and possibly bilateral, since activity in the human IC has been shown to be enhanced by selective topdown auditory attention (Rinne et al., 2008). Also, the lack of right IC activity might be due to the relative small sample size we employed here $(\mathrm{N}=12)$. Perhaps, a larger sample would yield significant activation also for the right IC.

The use of a condition controlling for neural refractoriness effects has been implemented in two fMRI studies (Opitz et al., 2005; Szycik et al., 2013), which compared physically identical stimuli embedded in either an oddball sequence or within randomly distributed equiprobable tones, and reported significant bilateral responses in the anterior portion of the $\mathrm{HG}$, a brain region that includes the primary auditory cortex. Overall, it seems that our DEV $>\mathrm{CON}$ contrast engaged a less distributed and spatially segregated cortical area with respect to these studies, as we reported a confined activation in the left anterior STG. Such a discrepancy might arise because in Opitz et al. (2005), participants were instructed to attend to the sounds, which might have led to an increased representation of the stimuli in the auditory cortex (Poghosyan and Ioannides, 2008; Petkov et al., 2004; Rinne et al., 2007). Additionally, in both studies the SOA was considerably slower $(600 \mathrm{~ms}$ and $1000 \mathrm{~ms})$ than the one adopted here, and deviant tones occurred with a lower probability (10\%), than in our study $(20 \%)$. Another reason might be related to the different type of stimulus, as the band-pass filtered noises here employed preferentially activate non-primary fields of the auditory cortex, while the pure tones used in Opitz et al. (2005) and in Szycik et al. (2013) produce a robust activation in the HG (Wessinger et al., 2001).

However, in line with our results, previous fMRI studies have reported a considerable smaller cortical area activated by the $\mathrm{DEV}>\mathrm{CON}$ than by the DEV $>$ STD contrast.

Although we reported a significant left superior temporal activity for $\mathrm{DEV}>\mathrm{CON}$, the lack of response in the auditory cortex (e.g. Heschl's gyrus) for this contrast merits attention. The employment of filtered noises delivered at particularly fast presentation rate $(150 \mathrm{~ms})$ makes our paradigm specifically suited for eliciting strong collicular responses. Thus, while the deviance detection mechanism based on release from adaptation-as encapsulated by the DEV $>$ STD condition-could be captured, the genuine deviance detection signal in the auditory cortex might have been interrupted by the quick occurrence of the next stimulus. That is, neurons of the auditory cortex might be more specialized in integrating stimulus features at larger time-scales.

Another aspect that deserves attention is that we have observed a larger response to the STD versus CON condition in both midbrain and thalamic structures (e.g., as depicted in Fig. 4e), although this difference did not reach significance, nor even a trend to significance (see Supplementary results). Nevertheless, one would expect to see a more adapted response to a repeated stimulation (i.e., our STD condition) rather than to sequences of randomly arranged stimuli differing in their physical features (i.e., our CON condition). This scenario might be in part due to an increased BOLD response for repeated stimuli, a phenomenon termed repetition enhancement (RE) (Segaert et al., 2013; Müller et al., 2013). Although the factors that determine a suppression or an increase of neuronal response to repeated stimulation have not yet been fully understood, RE has been repeatedly documented across sensory modalities (e.g., Doehrmann et al., 2010; Heinemann et al., 2011), and also using fMRI-adaptation auditory paradigms in the human IC (Chandrasekaran et al., 2012). Additionally, earlier magnetoencephalographic recordings have shown auditory RE effects for repeated sounds delivered at fast presentation rate (Loveless et al., 1989, 1996), similarly to the stimulus timing we employed our design. One 
difference between our random control condition and that adopted in earlier human EEG and fMRI studies, is that we captured brain activity across a fast sequence of 20 sounds, rather than to single stimuli. In order to more directly compare our results with those from previous studies, we performed a more strict analysis, where we compared the brain response elicited by each of the three subtypes of DEV conditions, to that elicited by the corresponding trains of the CON condition, matched for the physical position of the deviant stimulus (S2). This analysis confirmed significant responses in the IC and MGB in most of the contrasts of interest, thus strengthening our line of interpretation.

Whether auditory deviance detection in midbrain and thalamic structures emerges from sensorial input proceeding in a feedforward fashion, or it rather results from back-ward information traveling down the cortico-fugal pathway is still a matter of debate (cfr., Escera and Malmierca, 2014). To this regard, recent studies in rodents have demonstrated that reversible deactivation of auditory cortex does not prevent either collicular or thalamic SSA to occur (Antunes and Malmierca, 2011; Anderson and Malmierca, 2013). Additionally, SSA in the IC displays much shorter latencies than that in the auditory cortex (Malmierca et al., 2009). These findings strongly support the feed-forward account. However, the initial observation that auditory cortex is the first lemniscal station where SSA is robust and widespread (Ulanovsky et al., 2003) would rather substantiate the back-ward model. Likely, the two mechanisms are both at play and while SSA may originate subcortially, its amplitude and latency are possibly modulated by descending projections. The combination of high spatial resolution imaging with the implementation of causal connectivity modelling might shed light on the topic in animals as well as in humans.

It is worth noting that our data showing the existence of multiple mismatch detectors nested along the auditory pathway are well in line within predictive coding theories, which posit that our sensory systems encode statistical regularities by iteratively minimizing errors between top-down predictions and bottom-up sensory information, in hierarchically organized neural architectures (Rao and Ballard, 1999; Friston, 2005). Within this framework, an increased response to deviant stimuli represents the prediction error signal that is automatically fed to higher-level structures to adjust an internal model of stimulus representation (Baldeweg, 2006; Wacongne et al., 2012). Although our present paradigm was not specifically designed to test the efficiency of predictive coding models, future studies manipulating the number of standard stimulus repetitions and the degree of deviant stimuli salience, shall better address this topic.

Taken together, our data represent an important advance in understanding the functional organization of the auditory novelty system, by showing that the human IC and MGB belong to a larger network devoted to encode stimulus regularity and to detect acoustic violations. These results bridge the gap between previous studies conducted in animals and human electrophysiological as well as hemodynamic data. The specific functional role of the distinct signatures of change detection in higher order cognitive processes, such as speech comprehension or auditory object formation, shall be addressed in future studies performing additional manipulations of stimulus parameters, and combining auditory oddball paradigms with tasks probing short- and long-term learning.

\section{Acknowledgments}

This work was supported by the Spanish Ministry of Economy and Knowledge (PSI2012-37174) and the program Consolider-Ingenio 2010 (CSD2007-00012), the Catalan Government (SGR2014177), and the ICREA Academia Distinguished Professorship awarded to Carles Escera.

\section{References}

Alho, K., Grimm, S., Mateo-Leon, S., Costa-Faidella, J., Escera, C., 2012. Early processing of pitch in the human auditory system. Eur. J. Neurosci. 36, 2972-2978.
Althen, H., Grimm, S., Escera, C., 2011. Fast detection of unexpected sound intensity decrements as revealed by human evoked potentials. PLoS One 6, e28522.

Anderson, L.A., Christianson, G.B., Linden, J.F., 2009. Stimulus-specific adaptation occurs in the auditory thalamus. J. Neurosci. 29, 7359-7363.

Anderson, L.A., Malmierca, M.S., 2013. The effect of auditory cortex deactivation on stimulusspecific adaptation in the inferior colliculus of the rat. Eur. J. Neurosci. 37,52-62.

Antunes, F.M., Malmierca, M.S., 2011. Effect of auditory cortex deactivation on stimulus-specific adaptation in the medial geniculate body. J. Neurosci. 31, 17306-17316.

Antunes, F.M., Nelken, I., Covey, E., Malmierca, M.S., 2010. Stimulus-specific adaptation in the auditory thalamus of the anesthetized rat. PLoS One 5, e14071.

Ashburner, J., 2007. A fast diffeomorphic image registration algorithm. Neuroimage 38,95-113.

Baldeweg, T., 2006. Repetition effects to sounds: evidence for predictive coding in the auditory system. Trends Cogn. Sci. 10,93-94.

Bendixen, A., SanMiguel, I., Schröger, E., 2012. Early electrophysiological indicators for predictive processing in audition: a review. Int. J. Psychophysiol. 83, 120-131.

Bizley, J.K., Cohen, Y.E., 2013. The what, where and how of auditory-object perception. Nat. Rev. Neurosci. 14, 693-707.

Chandrasekaran, B., Kraus, N., Wong, P.C., 2012. Human inferior colliculus activity relates to individual differences in spoken language learning. J. Neurophysiol. 107, 1325-1336.

Deouell, L.Y., 2007. The frontal generator of the mismatch negativity revisited. J. Psychophysiol. 21, 188-203.

Doehrmann, O., Weigelt, S., Altmann, C.F., Kaiser, J., Naumer, M.J., 2010. Audiovisual functional magnetic resonance imaging adaptation reveals multisensory integration effects in object-related sensory cortices. J. Neurosci. 30, 3370-3379.

Doeller, C.F., Opitz, B., Mecklinger, A., Krick, C., Reith, W., Schröger, E., 2003. Prefrontal cortex involvement in preattentive auditory deviance detection: neuroimaging and electrophysiological evidence. Neuroimage 20, 1270-1282.

Escera, C., Alho, K., Winkler, I., Näätänen, R., 1998. Neural mechanisms of involuntary attention to acoustic novelty and change. J. Cogn. Neurosci. 10, 590-604.

Escera, C., Corral, M.J., 2007. Role of mismatch negativity and novelty-P3 in involuntary auditory attention. J. Psychophysiol. 21, 251-264.

Escera, C., Leung, S., Grimm, S., 2014. Deviance detection based on regularity encoding along the auditory hierarchy: electrophysiological evidence in humans. Brain Topogr. 27, 527-538.

Escera, C., Malmierca, M.S., 2014. The auditory novelty system: an attempt to integrate human and animal research. Psychophysiology 51, 111-123.

Friston, K., 2005. A theory of cortical responses. Philos. Trans. R. Soc. Lond. B Biol. Sci. 360 , 815-836.

Gao, P.P., Zhang, J.W., Cheng, J.S., Zhou, I.Y., Wu, E.X., 2014. The inferior colliculus is involved in deviant sound detection as revealed by BOLD fMRI. Neuroimage 91, 220-227.

Griffiths, T.D., Warren, J.D., 2004. What is an auditory object? Nat. Rev. Neurosci. 5, 887-892.

Grimm, S., Escera, C., 2012. Auditory deviance detection revisited: evidence fora hierarchical novelty system. Int. J. Psychophysiol 85,88-92.

Grimm, S., Escera, C., Slabu, L., Costa-Faidella, J., 2011. Electrophysiological evidence for the hierarchical organization of auditory change detection in the human brain. Psychophysiology 48, 377-384.

Grimm, S., Recasens, M., Althen, H., Escera, C., 2012. Ultrafast tracking of sound location changes as revealed by human auditory evoked potentials. Biol. Psychol. 89, 232-239.

Heinemann, L.V., Kaiser, J., Altmann, C.F., 2011. Auditory repetition enhancement at short interstimulus intervals for frequency-modulated tones. Brain Res. 1411, 65-75.

Ischebeck, A.K., Friederici, A.D., Alter, K., 2008. Processing prosodic boundaries in natural and hummed speech: an FMRI study. Cereb. Cortex 18, 541-552.

Jacobsen, T., Schröger, E., 2003. Measuring duration mismatch negativity. Clin. Neurophysiol. 114, 1133-1143.

King, C., McGee, T., Rubel, E.W., Nicol, T., Kraus, N., 1995. Acoustic features and acoustic changes are represented by different central pathways. Hear Res. 85, 45-52.

Kraus, N., McGee, T., Carrell, T., King, C., Littman, T., Nicol, T., 1994b. Discrimination of speech-like contrasts in the auditory thalamus and cortex. J. Acoust. Soc. Am. 96,2758-2768.

Kraus, N., McGee, T., Littman, T., Nicol, T., King, C., 1994a. Nonprimary auditory thalamic representation of acoustic change. J. Neurophysiol. 72, 1270-1277.

Lee, C.C., Sherman, S.M., 2011. On the classification of pathways in the auditory midbrain, thalamus, and cortex. Hear. Res. 276,79-87.

Leung, S., Cornella, M., Grimm, S., Escera, C., 2012. Is fast auditory change detection feature specific? An electrophysiological study in humans. Psychophysiology 49,933-942.

Logothetis, N.K., Pauls, J., Augath, M., Trinath, T., Oeltermann, A., 2001. Neurophysiological investigation of the basis of the fMRI signal. Nature 412, 150-157.

Loveless, N., Hari, R., Hamalainen, M., Tiihonen, J., 1989. Evoked responses of human auditory cortex may be enhanced by preceding stimuli. Electroencephalogr. Clin. Neurophysiol. 74, 217-227.

Loveless, N., Levanen, S., Jousmaki, V., Sams, M., Hari, R., 1996. Temporal integration in auditory sensory memory: neuromagnetic evidence. Electroencephalogr. Clin. Neurophysiol. 100, 220-228.

Maess, B., Jacobsen, T., Schröger, E., Friederici, A.D., 2007. Localizing pre-attentive auditory memory-based comparison: magnetic mismatch negativity to pitch change. Neuroimage 37, $561-571$

Maldjian, J.A., Laurienti, P.J., Kraft, R.A., Burdette, J.H., 2003. An automated method for neuroanatomic and cytoarchitectonic atlas-based interrogation of fMRI data sets. Neuroimage $19,1233-1239$.

Malmierca, M.S., Cristaudo, S., Pérez-González, D., Covey, E., 2009. Stimulus-specific adaptation in the inferior colliculus of the anesthetized rat. J. Neurosci. 29, 5483-5493.

May, P.J., Tiitinen, H., 2010. Mismatch negativity (MMN), the deviance-elicited auditorydeflection, explained. Psychophysiology 47,66-122. 
Mühlau, M., Rauschecker, J.P., Oestreicher, E., Gaser, C., Rottinger, M., Wohlschlager, A.M., Simon, F., Etgen, T., Conrad, B., Sander, D., 2006. Structural brain changes in tinnitus. Cereb. Cortex 16, 1283-1288.

Müller, N.G., Strumpf, H., Scholz, M., Baier, B., Melloni, L., 2013. Repetition suppression versus enhancement-it's quantity that matters. Cereb. Cortex 23, 315-322.

Näätänen, R., Jacobsen, T., Winkler, I., 2005. Memory-based or afferent processes in mismatch negativity (MMN): a review of the evidence. Psychophysiology 42, 25-32.

Näätänen, R., Gaillard, A.W., Mantysalo, S., 1978. Early selective-attention effect on evoked potential reinterpreted. Acta Psychol. (Amst) 42, 313-329.

Näätänen, R., Paavilainen, P., Rinne, T., Alho, K., 2007. The mismatch negativity (MMN) in basic research of central auditory processing: a review. Clin. Neurophysiol. 118, 2544-2590.

Opitz, B., Rinne, T., Mecklinger, A., von Cramon, D.Y., Schröger, E., 2002. Differential contribution of frontal and temporal cortices to auditory change detection: fMRI and ERP results. Neuroimage 15, 167-174.

Opitz, B., Schröger, E., von Cramon, D.Y., 2005. Sensory and cognitive mechanisms for preattentive change detection in auditory cortex. Eur. J. Neurosci. 21, 531-535.

Patel, C.R., Redhead, C., Cervi, A.L., Zhang, H., 2012. Neural sensitivity to novel sounds in the rat's dorsal cortex of the inferior colliculus as revealed by evoked localfield potentials. Hear. Res. 286,41-54.

Pérez-González, D., Malmierca, M.S., Covey, E., 2005. Novelty detector neurons in the mammalian auditory midbrain. Eur. J. Neurosci. 22, 2879-2885.

Petkov, C.I., Kang, X., Alho, K., Bertrand, O., Yund, E.W., Woods, D.L., 2004. Attentional modulation of human auditory cortex. Nat. Neurosci. 7, 658-663.

Poghosyan, V., Ioannides, A.A., 2008. Attention modulates earliest responses in the primary auditory and visual cortices. Neuron $58,802-813$

Rao, R.P., Ballard, D.H., 1999. Predictive coding in the visual cortex: a functional interpretation of some extra-classical receptive-field effects. Nat. Neurosci. 2, 79-87.

Recasens, M., Grimm, S., Capilla, A., Nowak, R., Escera, C., 2014. Two sequential processes of change detection in hierarchically ordered areas of the human auditory cortex. Cereb. Cortex 24, 143-153.

Richardson, B.D., Hancock, K.E., Caspary, D.M., 2013. Stimulus-specific adaptation in auditory thalamus of young and aged awake rats. J. Neurophysiol. 110, 1892-1902.

Rinne, T., Alho, K., Ilmoniemi, R.J., Virtanen, J., Nätänen, R., 2000. Separate time behaviors of the temporal and frontal mismatch negativity sources. Neuroimage 12,14-19.

Rinne, T., Balk, M.H., Koistinen, S., Autti, T., Alho, K., Sams, M., 2008. Auditory selective attention modulates activation of human inferior colliculus. J Neurophysiol 100, 3323-3327.

Rinne, T., Stecker, G.C., Kang, X., Yund, E.W., Herron, T.J., Woods, D.L., 2007. Attention modulates sound processing in human auditory cortex but not the inferior colliculus. Neuroreport 18, 1311-1314.

Sabri, M., Kareken, D.A., Dzemidzic, M., Lowe, M.J., Melara, R.D., 2004. Neural correlates of auditory sensory memory and automatic change detection. Neuroimage 21,69-74.

Schall, U., Johnston, P., Todd, J., Ward, P.B., Michie, P.T., 2003. Functional neuroanatomy of

auditory mismatch processing: an event-related fMRI study of duration-deviant oddballs.

Neuroimage 20, 729-736.

Schröger, E., Wolff, C., 1996. Mismatch response of the human brain to changes in sound location. Neuroreport 7, 3005-3008.

Segaert, K., Weber, K., de Lange, F.P., Petersson, K.M., Hagoort, P., 2013. The suppression of repetition enhancement: a review of fMRI studies. Neuropsychologia 51,59-66.

Slabu, L., Escera, C., Grimm, S., Costa-Faidella, J., 2010a. Early change detection in humans as revealed by auditory brainstem and middle-latency evoked potentials. Eur. J. Neurosci 32, $859-865$.

Slabu, L., Grimm, S., Escera, C., 2012. Novelty detection in the human auditory brainstem. J. Neurosci. 32, 1447-1452.

Slabu, L.M., 2010b. The effect of slice orientation on auditory FMRI at the level of the brainstem. Brain Topogr. 23, 301-310.

Sonnadara, R.R., Alain, C., Trainor, L.J., 2006. Occasional changes in sound location enhance middle latency evoked responses. Brain Res. 1076, 187-192.

Szycik, G.R., Stadler, J., Brechmann, A., Münte, T.F., 2013. Preattentive mechanisms of change detection in early auditory cortex: a 7 T fMRI study. Neuroscience $253,100-109$.

Taaseh, N., Yaron, A., Nelken, I., 2011. Stimulus-specific adaptation and deviance detection in the rat auditory cortex. PLoS One 6, e23369.

Tzourio-Mazoyer, N., Landeau, B., Papathanassiou, D., Crivello, F., Etard, O., Delcroix, N., Mazoyer, B., Joliot, M., 2002. Automated anatomical labeling of activations in SPM using a macroscopic anatomical parcellation of the MNI MRI single-subject brain. Neuroimage 15, 273-289.

Ulanovsky, N., Las, L., Nelken, I., 2003. Processing of low-probability sounds by cortical neurons. Nat. Neurosci. 6, 391-398.

Viswanathan, A., Freeman, R.D., 2007. Neurometabolic coupling in cerebral cortex reflects synaptic more than spiking activity. Nat. Neurosci. 10, 1308-1312.

von Kriegstein, K., Patterson, R.D., Griffiths, T.D., 2008. Task-dependent modulation of medial geniculate body is behaviorally relevant for speech recognition. Curr. Biol. 18, 1855-1859.

Wacongne, C., Changeux, J.P., Dehaene, S., 2012. A neuronal model of predictive coding accounting for the mismatch negativity. J. Neurosci. 32, 3665-3678.

Wessinger, C.M., VanMeter, J., Tian, B., Van Lare, J., Pekar, J., Rauschecker, J.P., 2001. Hierarchical organization of the human auditory cortex revealed by functional magnetic resonance imaging. J. Cogn. Neurosci. 13,1-7.

Winer, J.A., 2005. Three systems of descending projections to the inferior colliculus. In: Winer, J.A., Schreiner, C.E. (Eds.), The Inferior Colliculus. Springer, New York, pp. 231-247.

Winkler, I., Denham, S.L., Nelken, I., 2009. Modeling the auditory scene: predictive regularity representations and perceptual objects. Trends Cogn. Sci. 13, 532-540.

Woo, C.W., Krishnan, A., Wager, T.D., 2014. Cluster-extent based thresholding in fMRI analyses: pitfalls and recommendations. Neuroimage 91, 412-419. 